\title{
Effects of repeated subacute ruminal acidosis challenges on the adaptation of the rumen bacterial community in Holstein bulls
}

\author{
Rie Nagata, ${ }^{* 1}$ Yo-Han Kim, ${ }^{*} \dagger^{1}$ Akira Ohkubo, ${ }^{*}$ Shiro Kushibiki, $¥$ Toshihiro Ichijo, ${ }^{*}$ and Shigeru Sato*$\dagger^{2}$ \\ ${ }^{*}$ Cooperative Department of Veterinary Medicine, Faculty of Agriculture, Iwate University, Morioka, Iwate 020-8550, Japan \\ tUnited Graduate School of Veterinary Sciences, Gifu University, Gifu 501-1193, Japan \\ ¥National Institute of Livestock and Grassland Science, Tsukuba, Ibaraki 305-0901, Japan
}

\begin{abstract}
We investigated the effect of repeated subacute ruminal acidosis (SARA) challenges on the $\mathrm{pH}$, fermentative function, and bacterial community in the rumen. Four rumen-cannulated Holstein bulls were fed a high-forage diet for $7 \mathrm{~d}$ (HF period) followed by a high-grain diet for $7 \mathrm{~d}$ (HG period). Four SARA challenges were carried out consecutively (first, second, third, and fourth challenges). The ruminal $\mathrm{pH}$ was measured continuously during the experiment, and rumen fluid samples during the first to fourth challenges were collected at 0800,1400 , and $2000 \mathrm{~h}$ on the last days of each feeding period for analysis; volatile fatty acid components, $\mathrm{NH}_{3}-\mathrm{N}$, and lactic acid concentrations were measured. Bacterial community structure was analyzed at $0800 \mathrm{~h}$ during the first and fourth challenges on the last days of each period. The 24-h mean ruminal $\mathrm{pH}$ was decreased during the transition from high-forage to highgrain diet and tended to differ between the HF and HG periods. During the HG period, ruminal $\mathrm{pH}<5.6$ was maintained for a longer period in the first and second challenges (350 and $405 \mathrm{~min} / \mathrm{d}$, respectively) than in the third and fourth challenges (both $120 \mathrm{~min} / \mathrm{d}$ ). A marked increase in total volatile fatty acid and $\mathrm{NH}_{3}-\mathrm{N}$ concentrations during the $\mathrm{HG}$ period was observed in the later challenges. In addition, lower and higher proportions of acetic and butyric acids, respectively, were observed during the HG period than during the HF period. A total of 37 core bacterial genera were found in all samples; however, the relative abundance of several genera differed significantly between the HF and HG periods (Prevotella, Ruminococcus, Eubacterium, and Oscillibacter) and between the first and fourth chal-
\end{abstract}

Received September 20, 2017.

Accepted January 2, 2018.

${ }^{1}$ These authors contributed equally to this work.

${ }^{2}$ Corresponding author: sshigeru@iwate-u.ac.jp lenges (Eubacterium and unclassified Clostridiaceae). During the HG period, lower relative abundances of Prevotella, Eubacterium, and Oscillibacter and higher relative abundance of Ruminococcus were detected compared with during the HF period. The relative abundances of Eubacterium and unclassified Clostridiaceae were lower in the first challenge than in the fourth challenge. Bacterial diversity was greater during the $\mathrm{HF}$ period than during the HG period and was greater during the fourth challenge than during the first challenge. Interestingly, diversity indices during the HG period of the fourth challenge were higher than those during the HF period of the first challenge. These results suggested that rumen fermentation in Holstein cattle can adapt to repeated SARA challenges by minimizing the adverse changes in ruminal $\mathrm{pH}$. Moreover, the composition and diversity of the ruminal bacterial community may be affected by ruminal $\mathrm{pH}$ and vice versa.

Key words: bacterial community, cattle, repeated subacute ruminal acidosis challenge, rumen

\section{INTRODUCTION}

Around the beginning of the lactation period, dairy cows frequently undergo dietary transitions to match the energy requirement for milk production. The transition from a high-forage diet to a high-grain, highenergy diet results in greater VFA or lactate accumulation in the rumen (Owens et al., 1998; Rabelo et al., 2003), which contributes to increased risk of ruminal acidosis or SARA (Nagaraja and Titgemeyer, 2007). A condition characterized by ruminal $\mathrm{pH}<5.6$ (Gozho et al., 2005) or $<5.8$ (Penner et al., 2007) for an extended period, SARA may cause various health problems in dairy cows, such as feed intake depression, reduced fiber digestion, milk fat depression, diarrhea, laminitis, liver abscesses, increased production of bacterial endotoxins, and inflammation (Plaizier et al., 2008). Recently, highthroughput technologies have been used to evaluate the relationship between the incidence of ruminal acidosis 
or SARA and the rumen microbiota in cattle (Mao et al., 2013; Petri et al., 2013; Sato, 2016).

Repeated ruminal acidosis challenges affect ruminal $\mathrm{pH}$ and physiological behaviors, such as feeding, ruminating, and resting (Dohme et al., 2008; DeVries et al., 2009). As a result of the repeated acidosis challenges, depressed ruminal $\mathrm{pH}$, increased feeding and resting times, and decreased rumination times were observed in lactating dairy cows (Dohme et al., 2008; DeVries et al., 2009). During repeated acidosis challenges, less rumination was observed in cows at high risk for ruminal acidosis compared with cows at low risk for ruminal acidosis (DeVries et al., 2009). Furthermore, cows with high risk for acidosis exhibited lower ruminal $\mathrm{pH}$ and more severe ruminal acidosis compared with those at low risk for acidosis (Dohme et al., 2008). Therefore, dairy cattle may be able to adapt to repeated ruminal acidosis or SARA challenges by changing digestive behaviors. Furthermore, ruminal $\mathrm{pH}$ may be modulated by physiological responses.

The adaptation and recovery of rumen bacterial community structure and diversity were previously reported during single SARA and acidotic challenges (Hook et al., 2011; Petri et al., 2013). For instance, Hook et al. (2011) reported SARA-induced changes in ruminal bacterial density, diversity, and community structure, as analyzed by clone library construction and sequence analysis. Another study using Angus heifers revealed that the rumen core microbiome remains stable regardless of differences in diet or host genetics; several bacterial taxa were identified as part of the rumen core microbiome and were relatively unchanged when cattle were fed forage, mixed forage, or high grain and during acidotic challenges ( 4 and $12 \mathrm{~h}$ postfeeding) and recovery periods (Petri et al., 2013). Therefore, it was suggested that ruminal bacteria can adequately adapt to dietary or $\mathrm{pH}$ changes by altering bacterial composition and diversity (Hook et al., 2011; Petri et al., 2013).

Despite adaptation and recovery of rumen bacteria during a single acidosis or SARA challenge following high-grain feeding, it is known that dairy cows are fed concentrated diet every day during the lactation period to maximize milk production; the effect of repeated SARA challenges on the rumen bacterial community is largely unknown. Therefore, the objective of this study was to investigate the changes in ruminal $\mathrm{pH}$ and bacterial community adaptation to repeated SARA challenges induced by dietary transition from a high-forage diet to a high-grain diet. We hypothesized that repeated SARA challenges would induce changes in ruminal $\mathrm{pH}$ and fermentation ability, which in turn would affect bacterial community and diversity and vice versa.

\section{MATERIALS AND METHODS}

\section{Animals and Experimental Design}

All animals were cared for according to protocols approved by the Iwate University Laboratory Animal Care and Use Committee (A201401; Morioka, Japan). Four rumen-cannulated Holstein bulls $(192 \pm 12 \mathrm{~kg}$; $9.0 \pm 1.4$ mo of age) were used in this study. Following the 7-d high-forage adaptation period, all bulls were fed a high-forage diet for $7 \mathrm{~d}$ (HF period) and then a high-grain diet for $7 \mathrm{~d}$ (HG period). A SARA challenge was defined an HF period followed by a HG period, and each bull was exposed to 4 consecutive SARA challenges (first, second, third, and fourth challenges). It has been demonstrated that the ruminal $\mathrm{pH}$ can adapt to different diets within $7 \mathrm{~d}$ after dietary change, which prompted us to choose a repeat of a 7-d high-forage diet and 7-d high-grain diet (Sato, 2016). Furthermore, we wanted to investigate ruminal fermentation during repeated SARA in cattle whose fermentative conditions closely resemble those of cattle in the field. Bulls were fed mixed hay (orchard and timothy hay) during the HF period and a high-grain diet with a forage-toconcentrate ratio of 19:81 (DM basis) during the HG period (Table 1). Feed was supplied daily in 2 equal portions at 0800 and 1700 h. Daily DMI was recorded for individual animals throughout the experimental period, and it was confirmed that all feeds offered to animals were consumed. The amount and contents of the high-forage (control) and high-grain (SARA-inducing) diet chosen were based on our previous study (Kimura et al., 2012). The chemical compositions of the mixed hay and high-grain diet fed to the bulls are shown in Table 1.

Table 1. Composition of the high-forage diet and high-grain diet on percentage and DM bases

\begin{tabular}{lcc}
\hline Item & High-forage diet & High-grain diet \\
\hline Amount (\%) & 100 & 18.8 \\
Orchard and timothy hay & 0 & 60.9 \\
Concentrate & 0 & 20.3 \\
Corn flakes & 87.2 & 89.1 \\
DM & & \\
DM basis (\%) & 60.9 & 80.1 \\
TDN & 13 & 17 \\
CP & 1.3 & 3.6 \\
Crude fat & 40.5 & 13.8 \\
ADF & 68 & 26.7 \\
NDF & 0.5 & 0.8 \\
Calcium & 0.3 & 0.5 \\
Phosphate & & \\
\hline
\end{tabular}




\section{Sampling and Measurements}

Ruminal pH was measured continuously every $10 \mathrm{~min}$ throughout the experiment using a radio transmission system (YCOW-S; DKK-TOA Yamagata, Yamagata, Japan) as previously reported (Sato et al., 2012). A $\mathrm{pH}$ sensor was placed into the ventral sac of the rumen through the rumen fistula. Calibration was performed using standards at $\mathrm{pH} 4$ to 7 (DKK-TOA Yamagata) before, during (every $2 \mathrm{wk}$ ), and after the experiment, and no drift in $\mathrm{pH}$ was observed in the measurement during the experiment period. Fluid samples from the first, second, third, and fourth challenges were collected from the rumen at 0800,1400 , and $2000 \mathrm{~h}$ on the last days of the HF and HG periods. The collected samples were immediately filtered through 2 layers of cheesecloth and were stored at $-80^{\circ} \mathrm{C}$ until usage. For VFA analysis, $1 \mathrm{~mL}$ of $25 \% \mathrm{HO}_{3} \mathrm{P}$ in $3 \mathrm{~N} \mathrm{H}_{2} \mathrm{SO}_{4}$ was added to $5 \mathrm{~mL}$ of rumen fluid. Total VFA and VFA components (i.e., acetic acid, propionic acid, and butyric acid) were separated and quantified by GC (model 135, Hitachi, Tokyo, Japan) using a packed glass column (Thermon-3000, 3\%) with a Shimalite TPA 60-80 mesh support (Shinwa Chemical Industries Ltd., Kyoto, Japan). For lactic acid analysis, fluid samples were centrifuged at $2,000 \times g$ for $15 \mathrm{~min}$ at $4^{\circ} \mathrm{C}$, and concentration of lactic acid in the supernatant was determined using a commercially available kit (F-kit; D-lactate/L-lactate, J. K. International Co., Tokyo, Japan). To measure $\mathrm{NH}_{3}-\mathrm{N}$ concentration, fluid samples were analyzed using the steam distillation method with an $\mathrm{NH}_{3}-\mathrm{N}$ analyzer (Kjeltec Auto Sampler System 1035 Analyzer, Tecator Inc., Höganäs, Sweden).

\section{DNA Isolation}

Total bacterial DNA was extracted from rumen fluid samples collected at $0800 \mathrm{~h}$ during the first and fourth challenges on the last days of the $\mathrm{HF}$ and $\mathrm{HG}$ periods as previously described (Kim et al., 2016). The rumen fluid sample was incubated with $750 \mu \mathrm{g} / \mathrm{mL}$ of lysozyme (Sigma-Aldrich Co., St. Louis, MO) at $37^{\circ} \mathrm{C}$ for $90 \mathrm{~min}$. This was followed by the addition of $10 \mu \mathrm{L}$ of purified achromopeptidase (Wako Pure Chemical Industries Ltd., Osaka, Japan) at a concentration of $10,000 \mathrm{U} / \mathrm{mL}$, and the mixture was incubated at $37^{\circ} \mathrm{C}$ for $30 \mathrm{~min}$. The suspension was treated with $60 \mu \mathrm{L}$ of $1 \%$ SDS and $1 \mathrm{mg} / \mathrm{mL}$ of proteinase $\mathrm{K}$ (Merck Japan Ltd., Tokyo, Japan) and was incubated at $55^{\circ} \mathrm{C}$ for 5 min. The lysate was treated with phenol/chloroform/ isoamyl alcohol (Wako Pure Chemical Industries Ltd.) and chloroform (Life Technologies Japan Ltd., Tokyo, Japan). The DNA was precipitated with $5 \mathrm{M} \mathrm{NaCl}$ and
$100 \%$ ethanol, followed by centrifugation at $21,900 \times g$ for $15 \mathrm{~min}$ at $4^{\circ} \mathrm{C}$. The DNA pellet was rinsed with $70 \%$ ethanol, dried, and dissolved in Tris-hydrochloride buffer. Purified DNA was quantified using a Biospec-nano (Shimadzu Biotech, Kyoto, Japan) and was stored at $-80^{\circ} \mathrm{C}$ until further analysis.

\section{DNA Pyrosequencing}

The $\mathrm{V} 1 / \mathrm{V} 2$ region of the $16 \mathrm{~S}$ rRNA gene was amplified using a forward primer (5'-CCATCTCATCCCTGCGTGTCTCCGACTCAGNNNNNNNNNNAGRGTTTGATYMTGGCTCAG-3') that contained 454 primer A, a 10-bp barcode sequence unique for each sample (indicated as N), and 27Fmod (5'-AGRGTTTGATYMTGGCTCAG), in which the third base, A, in the original primer $27 \mathrm{~F}$ was changed to $\mathrm{R}$. The reverse primer (5'-CCTATCCCCTGTGTGCCTTGGCAGTCTCAGTGCTGCCTCCCGTAGGAGT-3') contained 454 primer $\mathrm{B}$ and the reverse primer 338R (5'-TGCTGCCTCCCGTAGGAGT). Amplified products (approximately $370 \mathrm{bp}$ ) were confirmed using agarose gel electrophoresis. They were then purified using AMPure XP magnetic purification beads (Beckman Coulter Inc., Brea, CA) and were quantified using the Quant-iT PicoGreen dsDNA assay kit (Life Technologies Japan Ltd.). Mixed samples were prepared by pooling approximately equal amounts of PCR amplicons from each sample, which were subjected to 454 GS Junior (Roche Applied Science, Indianapolis, IN) sequencing, following the manufacturer's instructions. The sequencing data were deposited into the Sequence Read Archive of the National Center for Biotechnology Information and can be accessed via the accession number SRP115745 (https://submit.ncbi.nlm.nih.gov/subs/sra/).

\section{Pyrosequencing Data Analysis}

All pyrosequencing reads were filtered according to procedures outlined by Kim et al. (2013), who developed an analysis pipeline for barcoded 454 pyrosequencing of 27Fmod/338R-amplified PCR products in the V1/ $\mathrm{V} 2$ region. Pyrosequencing reads were assigned to each sample based on the barcode sequence information. The resulting sequences that did not have PCR primer sequences at both sequence termini and those with average quality values $>25$ were filtered out. Chimeras with $>90 \%$ similarity to reference sequences in the database, as analyzed by BLAST (https://blast.ncbi.nlm .nih.gov/Blast.cgi), were removed. Finally, filter-passed reads were obtained for further analysis by trimming off both primer sequences. For operational taxonomic unit (OTU) analysis, 16S reads were clustered using a $96 \%$ 
pairwise identity cutoff in the UCLUST program (www .drive5.com). Representative sequences for each OTU were assigned to bacterial species based on BLAST searches with a $96 \%$ pairwise identity cutoff against the $16 \mathrm{~S}$ rRNA gene sequence database; sequence comparisons were carried out using the Ribosomal Database Project tools (version 10.27; http://rdp.cme.msu.edu/ ) and against a reference genome database constructed from genome sequences collected from GenBank (ftp:// ftp.ncbi.nih.gov/GenBank/; Nov. 2013).

After trimming off both primer sequences, a total of 138,555 filter-passed sequences were obtained from the analysis pipeline (Kim et al., 2013). Obtained filter-passed reads were processed using the MOTHUR program (version 1.35, University of Michigan; http:// www.mothur.org/wiki/; Schloss et al., 2009), and all samples were standardized by random subsampling (4,623 sequences/sample) using the "sub.sample" command to generate rarefaction curves. The abundancebased coverage estimator, Chao1 richness estimator, Shannon diversity index, and the Good's coverage were calculated using MOTHUR following the 454 standard operating procedure (Schloss et al., 2011). To obtain a nonredundant set of sequences, unique sequences were determined and were used to align against the SILVA reference alignment database (SSURef release 119; Pruesse et al., 2007); chimera were removed using the chimera.uchime program (http://drive5.com/uchime); sequences identified as being of eukaryotic origin were removed; candidate sequences were screened and preclustered to eliminate outliers; and a distance matrix was generated from the resulting sequences. Sequences were clustered into OTU with a cutoff value of $97 \%$ similarity. The rarefaction curve was generated at the 97\% similarity level, which was calculated by distancebased OTU (Schloss et al., 2011). The "summary. single" command was used for calculation of the Good's coverage, Chao1, abundance-based coverage estimator, and Shannon diversity index. The unweighted UniFrac distance method (Lozupone and Knight, 2005) was used to perform principal coordinates analyses with all OTU.

\section{Statistical Analysis}

The 24-h mean ruminal $\mathrm{pH}$ data during all challenges were categorized as follows: minimum, mean, maximum, duration of time where $\mathrm{pH}$ was $<5.6$, and area under $\mathrm{pH}$ 5.6. Total VFA, $\mathrm{NH}_{3}-\mathrm{N}$, and lactic acid concentrations, proportions of individual VFA, and ruminal ratios of acetic acid to propionic acid in the first, second, third, and fourth challenges were summarized at 0800,1400 , and $2000 \mathrm{~h}$ on the last days of the HF and HG periods. The relative abundance of bacterial phyla and genera, as well as indices of bacterial diversity, were summarized at $0800 \mathrm{~h}$ during the first and fourth challenges on the last days of the $\mathrm{HF}$ and $\mathrm{HG}$ periods. The normality of the distribution of variables was assessed using the Shapiro-Wilk test.

One-way repeated-measures ANOVA, followed by Tukey's multiple comparison method, was used to determine differences in total $\mathrm{VFA}, \mathrm{NH}_{3} \mathrm{~N}$, lactic acid concentrations, proportions of individual VFA, and ruminal ratios of acetic acid to propionic acid. Twoway repeated-measures ANOVA, followed by multiple testing false discovery rate, was used to determine differences in ruminal $\mathrm{pH}$, the duration of time where $\mathrm{pH}$ was $<5.6$, the area under $\mathrm{pH} 5.6$, the relative abundance of bacterial phyla and genera, and bacterial diversity indices. Two-way repeated-measures ANOVA was used to examine main effects of period, challenge, and their interactions as well as random effects of the animal. Bacterial phyla and genera with relative abundances below our threshold $(>1 \%)$ were excluded from statistical analysis.

Heat maps with double dendrograms were constructed using the gplots package in the $\mathrm{R}$ project software version 3.3.2 (http://www.r-project.org; R Foundation for Statistical Computing, Vienna, Austria) with relative abundance data. All numerical data were expressed as means \pm standard error and were analyzed using Prism version 7.01 software (GraphPad Software Inc., La Jolla, CA). Significant differences were set as $P<$ 0.05 , and trends that suggested possible significance were set at $P<0.10$.

\section{RESULTS}

\section{Ruminal $\mathrm{pH}$ and VFA}

The 24-h minimum ruminal $\mathrm{pH}$ was found to differ significantly $(P<0.05)$ between the 2 feeding periods, whereas the mean and maximum ruminal $\mathrm{pH}$ showed a trend for difference $(P=0.060$ and 0.010 , respectively) between the 2 periods (Table 2). During the HF period in every challenge, $\mathrm{pH}$ values were $>5.6$. Results indicated that the duration of time where $\mathrm{pH}$ was $<5.6$ during the HG period was longer in the second challenge than in the first challenge. During the HG period, $\mathrm{pH}<5.6$ was observed for an extended period in the first and second challenges $(350 \pm 171$ and 405 $\pm 260 \mathrm{~min} / \mathrm{d}$, respectively); duration of time where ruminal $\mathrm{pH}$ was $<5.6$ in the HG period was $120 \pm 81$ and $120 \pm 75 \mathrm{~min} / \mathrm{d}$ in the third and fourth challenges, respectively. Diurnal changes in the 1-h mean ruminal $\mathrm{pH}$ were more pronounced during the HG period than during the HF period. In addition, drastic fluctuations in the 1-h mean ruminal $\mathrm{pH}$ induced by transition from 


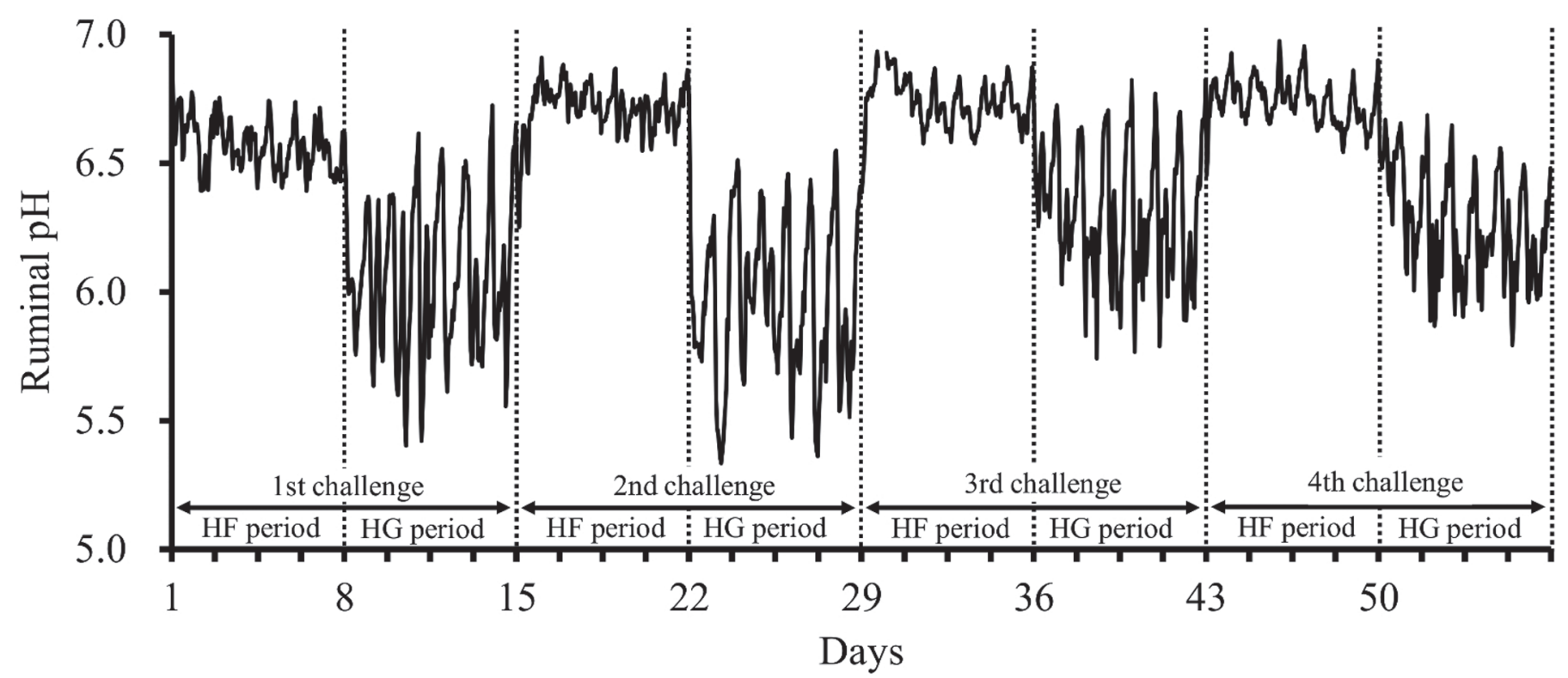

Figure 1. Changes in the 1-h mean ruminal $\mathrm{pH}$ in the first, second, third, and fourth SARA challenges. Cattle were fed a high-forage diet for $7 \mathrm{~d}$ (HF period) and then a high-grain diet for $7 \mathrm{~d}$ (HG period). A SARA challenge was defined as an HF period followed by a HG period, and 4 SARA challenges were carried out consecutively (first, second, third, and fourth challenges).

high-forage diet to high-grain diet during the first 2 challenges were mitigated in later challenges (Figure 1). The effect of challenge and the interaction between feeding period and challenge were not significant for ruminal $\mathrm{pH}$, duration of time where $\mathrm{pH}$ was $<5.6$, and area under $\mathrm{pH}$ 5.6.

Total VFA concentration in the third and fourth challenges was significantly $(P<0.05)$ higher than the first challenge during both periods, and that in the second and fourth challenges was significantly $(P$ $<0.05)$ higher during the HG period than during the $\mathrm{HF}$ period (Figure 2). The $\mathrm{NH}_{3}-\mathrm{N}$ concentration in the second and third challenges was significantly $(P$ $<0.05)$ higher than the first challenge during the HF and HG periods. A lower proportion of acetic acid and higher proportions of butyric acid and other VFA (ex- cluding the proportions of acetic acid, propionic acid, and butyric acid) were observed during the HG period compared with the HF period. No significant difference was observed in lactic acid concentration, proportion of propionic acid, and ratios of acetic acid to propionic acid.

\section{Bacterial Abundance}

A total of 19 bacterial phyla and 1 candidate phylum were identified within ruminal bacteria. Of the major phyla, Firmicutes (43.1\%), Bacteroidetes (26.0\%), and Actinobacteria (2.9\%) were found to be the most abundant. The remaining phyla exhibited low relative abundances of $<1 \%$. A total of 360 bacterial genera were identified; out of these, the relative abundance of

Table 2. Changes in the 24-h mean ruminal $\mathrm{pH}$, duration of time where $\mathrm{pH}$ was $<5.6$, and area under $\mathrm{pH} 5.6$ during the high-forage diet period (HF period; $\mathrm{n}=4$ ) and the high-grain diet period (HG period; $\mathrm{n}=4$ ) in SARA challenges $1,2,3$, and 4

\begin{tabular}{|c|c|c|c|c|c|c|c|c|c|c|c|c|}
\hline \multirow[b]{2}{*}{ Item } & \multicolumn{4}{|c|}{ HF period } & \multicolumn{4}{|c|}{ HG period } & \multirow[b]{2}{*}{ SEM } & \multicolumn{3}{|c|}{$P$-value } \\
\hline & 1 & 2 & 3 & 4 & 1 & 2 & 3 & 4 & & $\begin{array}{l}\text { Period } \\
(\mathrm{P})\end{array}$ & $\begin{array}{l}\text { Challenge } \\
\text { (C) }\end{array}$ & $\mathrm{P} \times \mathrm{C}$ \\
\hline Minimum & 6.30 & 6.48 & 6.47 & 6.45 & 5.27 & 5.18 & 5.49 & 5.63 & 0.11 & 0.016 & 0.121 & 0.320 \\
\hline Mean & 6.52 & 6.72 & 6.72 & 6.68 & 6.10 & 5.91 & 6.25 & 6.15 & 0.07 & 0.060 & 0.262 & 0.244 \\
\hline Maximum & 6.84 & 6.97 & 6.96 & 6.88 & 6.84 & 6.64 & 6.83 & 6.54 & 0.04 & 0.100 & 0.124 & 0.249 \\
\hline $\begin{array}{l}\text { Area under curve } \\
\mathrm{pH}<5.6, \mathrm{pH} \times \min / \mathrm{d}\end{array}$ & 0 & 0 & 0 & 0 & 13.3 & 24.3 & 0.6 & 1.8 & 2.98 & 0.182 & 0.477 & 0.477 \\
\hline
\end{tabular}


347 genera composed $<1 \%$ of total bacterial sequences. Analysis of genus-level composition and abundance of the core bacterial community identified 37 genera that were shared by $100 \%$ of the samples. Prevotella (17.7\%), Ruminococcus (10.3\%), and Clostridium (5.4\%) were the most abundant bacterial genera with respect to proportion of total sequence. At the OTU level, the total of richness of all groups was determined to be 6,903 (Supplemental Figure S1; https://doi.org/10.3168/jds .2017-13859). The number of OTU in the first challenge was 2,931 and 1,652 during the HF and HG periods, respectively; the OTU number in the fourth challenge was 3,642 and 2,722 during the HF and HG periods, respectively. As demonstrated by a Venn diagram, the number of shared OTU between the HF and HG periods was higher in the fourth challenge (1,327 OTU) compared with the first challenge (816 OTU).
At the phylum level, the relative abundance of Firmicutes was significantly $(P<0.05)$ different between the HF and HG periods, whereas that of Bacteroidetes showed a trend toward difference $(P=0.058)$ between the 2 feeding periods. The relative abundance of Firmicutes was lower during the HF period, and the relative abundance of Bacteroidetes was higher during the HG period (Figure 3). The relative abundance of Actinobacteria tended to differ $(P=0.090)$ between the first and fourth challenges. The relative abundance of Firmicutes and Actinobacteria was higher and the relative abundance of Bacteroidetes was lower in the first challenge compared with the fourth challenge. No significant interactions between the effect of feeding period and challenge were detected concerning relative abundances of Firmicutes, Bacteroidetes, and Actinobacteria.
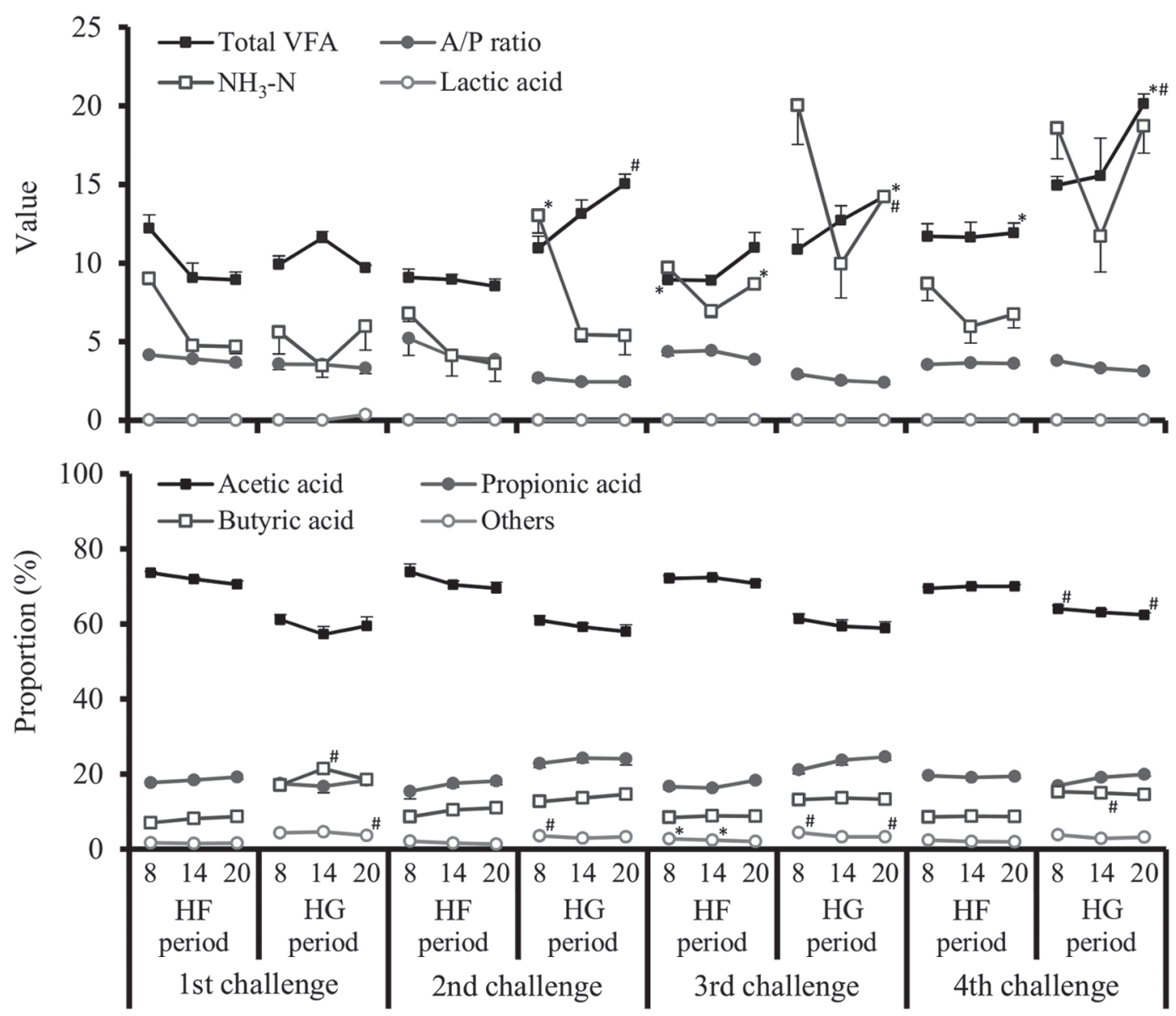

Figure 2. Diurnal changes in ruminal total VFA (mmol/dL), $\mathrm{NH}_{3}-\mathrm{N}(\mathrm{mg} / \mathrm{dL})$, and lactic acid $(\mathrm{g} / \mathrm{L})$ concentrations, ratios of acetic acid to propionic acid, and proportions of individual VFA in the first, second, third, and fourth SARA challenges. Cattle were fed a high-forage diet for $7 \mathrm{~d}$ (HF period) and then a high-grain diet for $7 \mathrm{~d}$ (HG period). A SARA challenge was defined as an HF period followed by a HG period, and 4 SARA challenges were carried out consecutively (first, second, third, and fourth challenges). The numbers 8,14 , and 20 indicate time of the last day of each period. Values represent mean \pm SE. *Significant difference $(P<0.05)$ compared with the first challenge at the same period and time point. ${ }^{\#}$ Significant difference $(P<0.05)$ between the HF and HG periods at the same challenge and time point. 
At the genus level, the relative abundance of Prevotella, Ruminococcus, Eubacterium, and Oscillibacter differed significantly $(P<0.05)$ between the HF and HG periods. The relative abundance of Prevotella, Eubacterium, and Oscillibacter was higher during the HF period, whereas the relative abundance of Ruminococcus was higher during the HG period (Figure 4). The relative abundance of Clostridium and Succiniclasticum exhibited a trend for difference $(P=0.097$ and 0.052 , respectively) between the 2 periods. The relative abundance of Eubacterium and unclassified Clostridiaceae was significantly $(P<0.05)$ different between the first and fourth challenges; lower relative abundance of these 2 genera was detected during the first challenge compared with the fourth challenge. The relative abundance of Clostridium and Oscillibacter tended to differ $(P=0.074$ and 0.084 , respectively) between the 2 challenges. In addition, significant interaction between feeding period and challenge was detected $(P<0.05)$, which affected the relative abundance of Clostridium, Eubacterium, and Oscillibacter. No significant difference was observed in the relative abundance of Clostridium, whereas the relative abundance of Eubacterium during the HF period in the fourth challenge was significantly $(P<0.05)$ higher compared with the first challenge as well as during the first and fourth challenges in the HG period. The relative abundance of Oscillibacter in the first challenge during the HF period was significantly $(P<0.05)$ higher compared with the first and fourth challenges during the HG period.

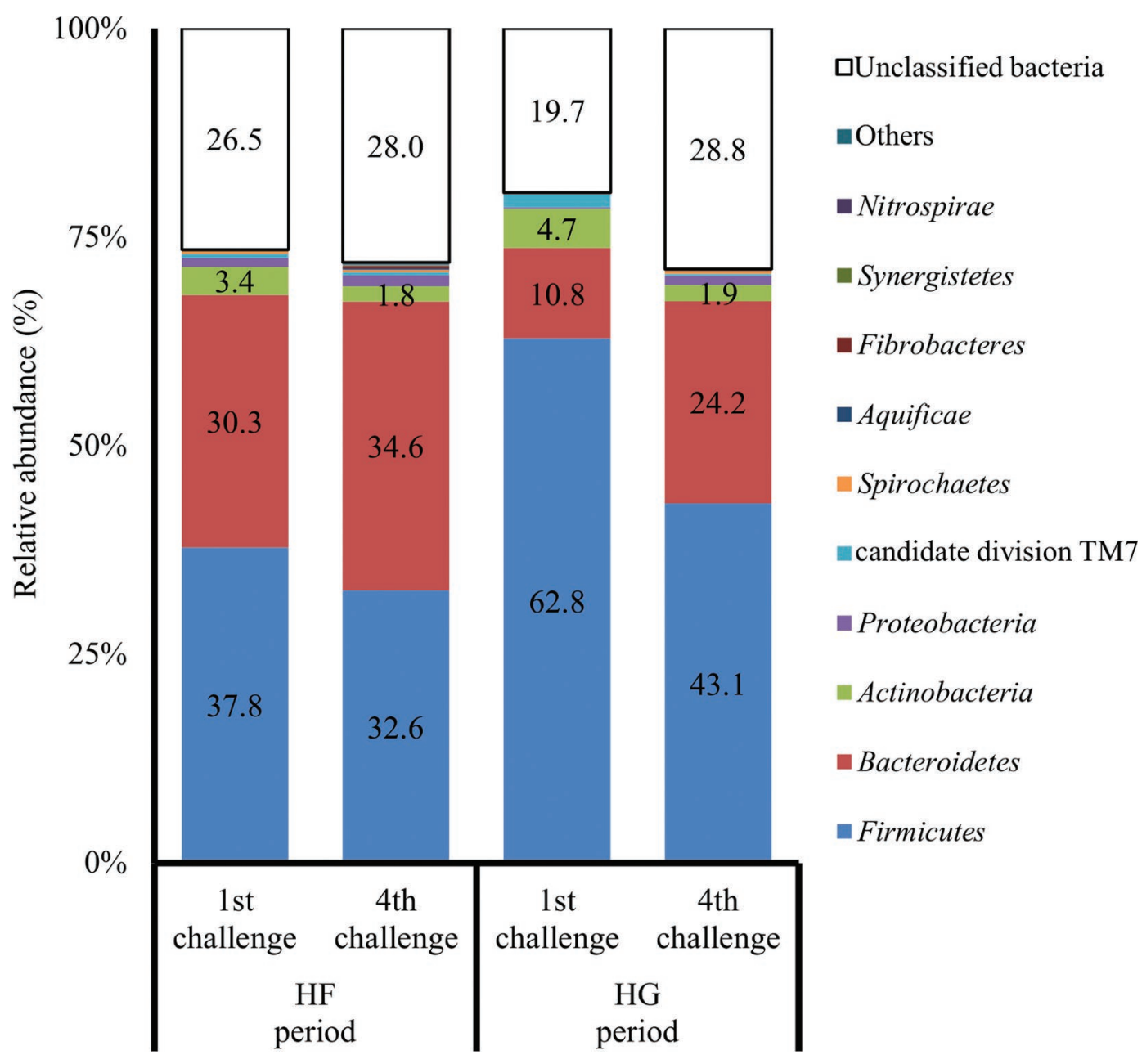

Figure 3. Relative abundance (\% of total sequences) of the major rumen fluid bacterial phyla in the first and fourth SARA challenges. Cattle were fed a high-forage diet for $7 \mathrm{~d}$ (HF period) and then a high-grain diet for $7 \mathrm{~d}$ (HG period). Four SARA challenges were carried out consecutively (first, second, third, and fourth challenges). The numbers within each bar indicate the percentage of each bacterial phylum. Color version available online. 


\section{Bacterial Diversity Analysis}

An average coverage was $87.6 \pm 1.2 \%($ mean $\pm \mathrm{SE})$ in our study. The rarefaction curves generated at $97 \%$ similarity level demonstrated that the HF period had higher bacterial diversity than the HG period regardless of the challenges (Supplemental Figure S2; https:// doi.org/10.3168/jds.2017-13859). Bacterial diversity

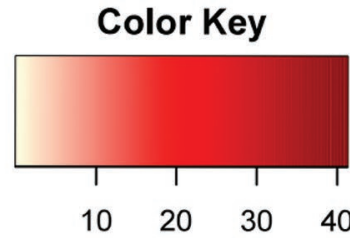

Relative abundance (\%)

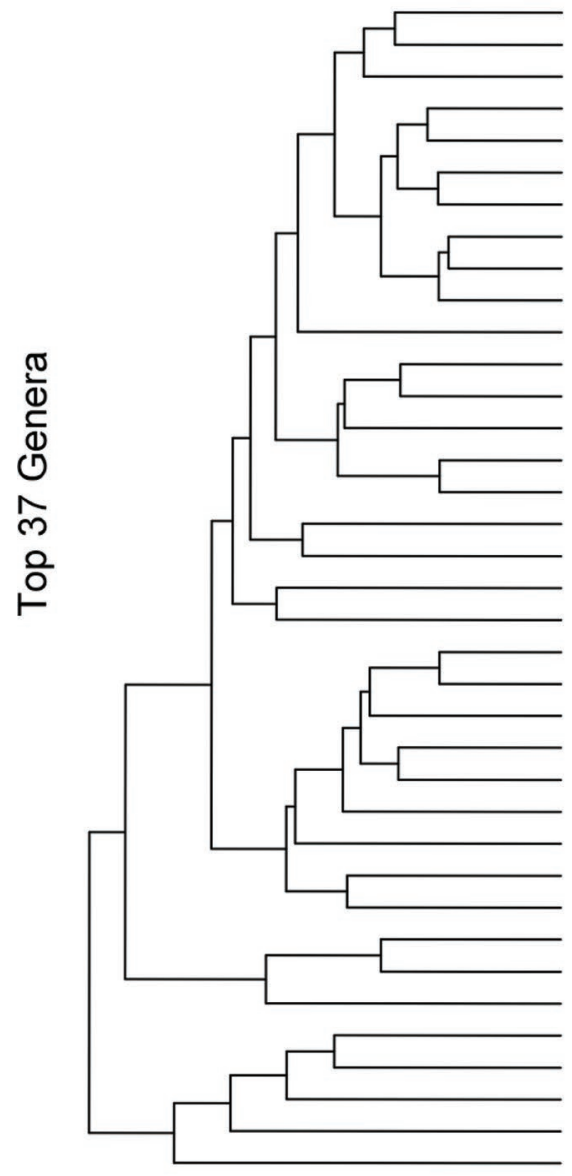

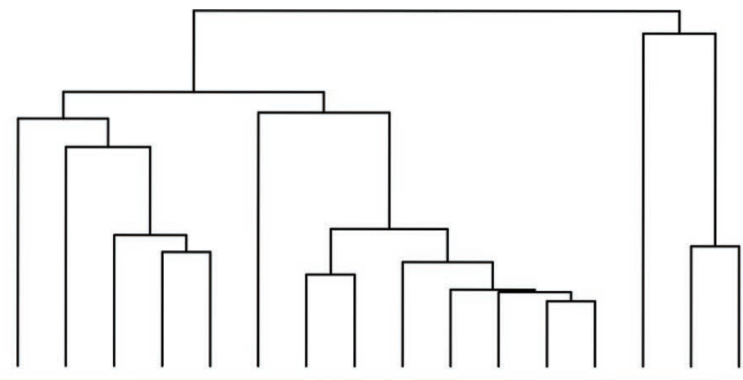

Thermicanus

Selenomonas

Christensenella

Blautia

Syntrophococcus

Coprococcus

unclassified Peptostreptococcaceae

Anaerovorax

Faecalibacterium

Mogibacterium

Moryella

candidate division TM7

Solobacterium

Olsenella

Roseburia

Oribacterium

Lachnobacterium

Actinotalea

Bulleidia

Dorea

Acetivibrio

unclassified Clostridiales

unclassified Lachnospiraceae

Pseudobutyrivibrio

Succiniclasticum

Oscillibacter

Streptococcus

unclassified Clostridiaceae

Eubacterium

Atopobium

Lactobacillus

Schwartzia

Butyrivibrio

Clostridium

Ruminococcus

Barnesiella

Prevotella

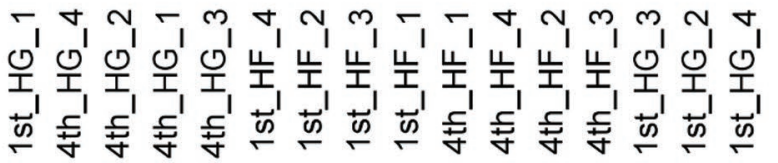

\section{Individual Cattle}

Figure 4. Heat map with double dendrogram of a hierarchical clustering analysis consisting of the 37 genera shared by $100 \%$ of the samples. Cattle were fed a high-forage diet for $7 \mathrm{~d}$ (HF period) and then a high-grain diet for $7 \mathrm{~d}$ (HG period). Four SARA challenges were carried out consecutively (first, second, third, and fourth). 1st and 4th represent the first and fourth challenges, respectively, and HF and HG represent the HF period and HG period, respectively. The number indicates the individual cattle number. Color version available online. 

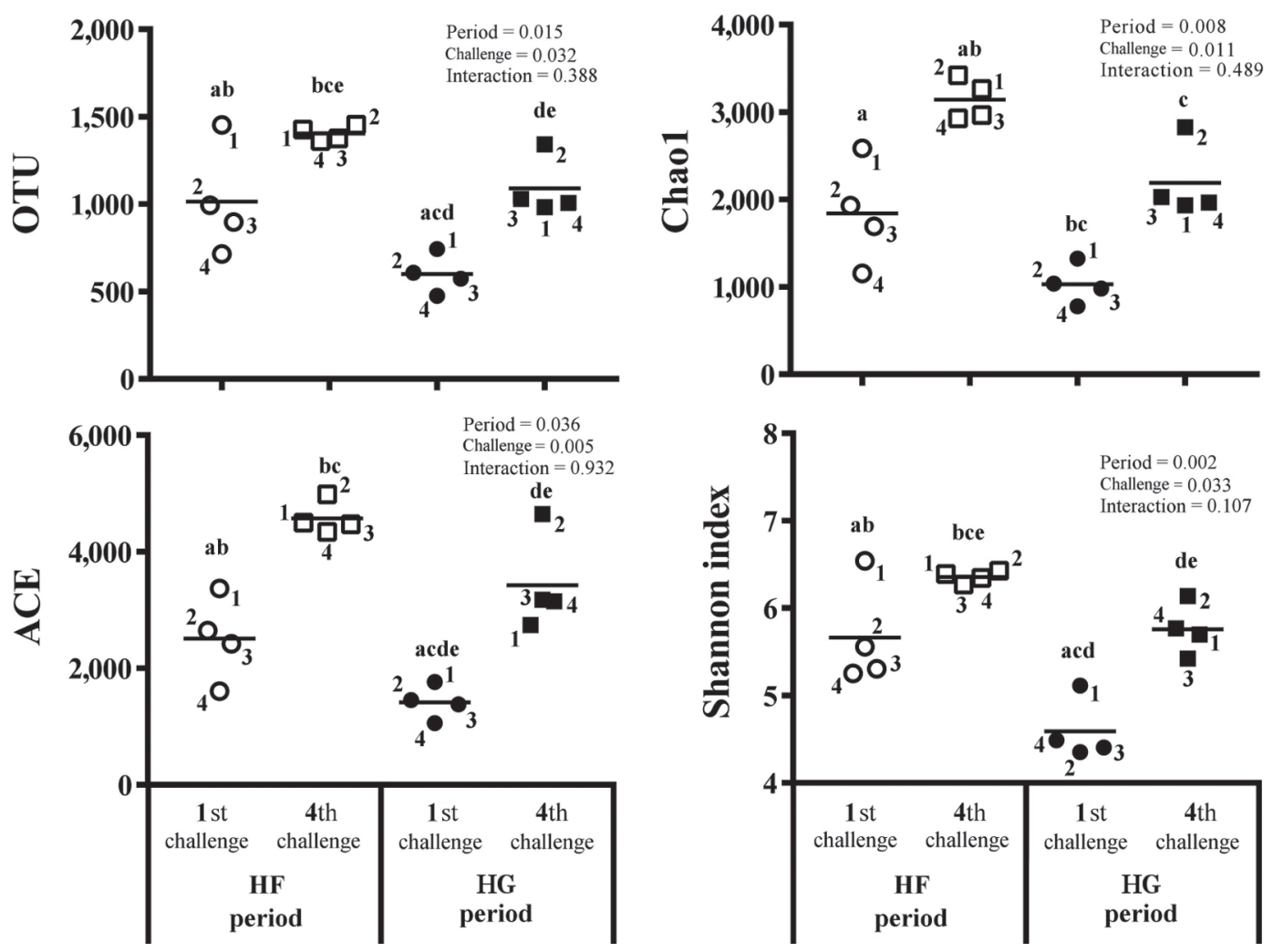

Figure 5. Column scatter plots of bacterial diversity indices calculated from 454 pyrosequencing data in the first and fourth SARA challenges. Cattle were fed a high-forage diet for $7 \mathrm{~d}$ (HF period) and then a high-grain diet for $7 \mathrm{~d}$ (HG period). Four SARA challenges were carried out consecutively (first, second, third, and fourth challenges). The number indicates the individual cattle number. Values with the same letters (a-e) significantly differ $(P<0.05)$. OTU $=$ operational taxonomic unit; ACE = abundance-based coverage estimator.

indices during both feeding periods were significantly $(P<0.05)$ higher in the fourth challenge than in the first challenge; OTU and Shannon index during the HF period were also significantly $(P<0.05)$ higher than during the HG period regardless of the challenges (Figure 5). No interaction was observed between the feeding period and the challenge in terms of ruminal bacterial diversity indices such as OTU, abundance-based coverage estimator, Chao1, and the Shannon index. Principal coordinates analysis plots showed close similarity within and between challenges during the HF period, whereas those during the HG period showed dispersed distribution within each challenge and between the first and fourth challenges (principal components $1+2=$ $31.9 \%$; Figure 6).

\section{DISCUSSION}

This study was carried out to investigate the effect of repeated SARA challenges on the $\mathrm{pH}$, bacterial communities, and bacterial diversity in rumen fluids. Solidassociated bacterial community was different from liquid-associated bacterial community in bacterial mass
(Zebeli et al., 2008) and populations (Metzler-Zebeli et al., 2015) in the rumen. Furthermore, Petri et al. (2017) reported that the in situ fiber-adherent rumen bacterial community was significantly affected by SARA condition in bacterial diversity, relative abundance, and predicted functional pathway analysis, whereas the solid-associated bacterial community was not examined in this study. Sato (2016) previously reported that changes in ruminal $\mathrm{pH}$ typically respond to different types of diets within a week following the dietary transition. Our study showed that SARA was successfully induced in the first and second challenges during the 1-wk interval of repeated SARA challenges. However, the severity of SARA was alleviated in the third and fourth challenges. During the HG period, we found that the period during which $\mathrm{pH}$ was $<5.6$ was longer in the second challenge than in the first challenge. According to Pourazad et al. (2016), transient model dairy cattle that had a 1-wk break of forage-only diet after 1 wk of SARA challenge exhibited ruminal $\mathrm{pH}<5.5$, 5.8 , and 6.0 for a longer period during the postbreak SARA challenge than during the prebreak SARA challenge. Furthermore, transient SARA induced more se- 


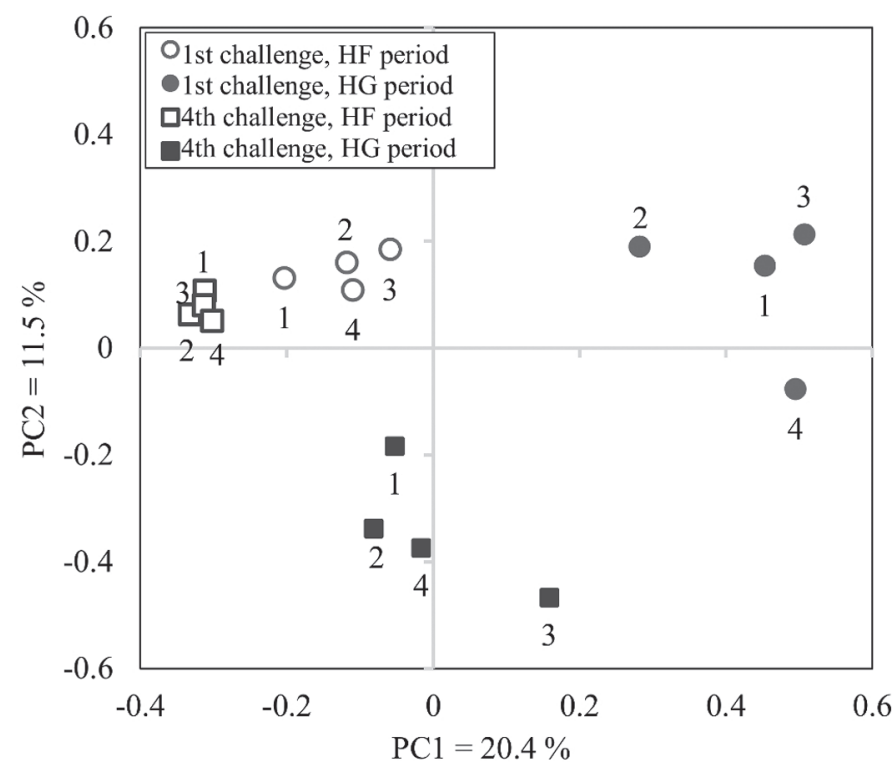

Figure 6. Principal coordinates analysis plots generated from the 454 pyrosequencing data in the first and fourth SARA challenges. Cattle were fed a high-forage diet for $7 \mathrm{~d}$ (HF period) and then a highgrain diet for $7 \mathrm{~d}$ (HG period). Four SARA challenges were carried out consecutively (first, second, third, and fourth challenges). PC1 and PC2 represent principal components 1 and 2, respectively. The number indicates the individual cattle number.

vere SARA than the 4-wk continuous SARA challenge in dairy cattle (Pourazad et al., 2016). In our study, we conducted repeated SARA challenges 4 times and found that drastic changes in ruminal $\mathrm{pH}$ during the HG period were mitigated during the later challenges. This may have contributed to the reduced differences in ruminal $\mathrm{pH}$, the time duration where ruminal $\mathrm{pH}$ was $<5.6$, and the area under $\mathrm{pH} 5.6$. However, the mechanisms underlying adaptation of ruminal $\mathrm{pH}$ to repeated SARA challenges could not be identified because feeding behavior and physiological responses were not observed in this study.

During 4 consecutive SARA challenges, the adaptation of rumen fermentation was identified simultaneously with that of ruminal $\mathrm{pH}$. In this study, lower and higher proportions of acetic and butyric acids, respectively, were identified during the HG period; these results were consistent with the general features of a high-grain diet (Bevans et al., 2005; Sato, 2016). Interestingly, we identified a concurrent increase in total VFA and $\mathrm{NH}_{3}-\mathrm{N}$ concentrations during the $\mathrm{HG}$ period of the later challenges, although it may denote an increased risk to VFA accumulation in SARA (Burrin and Britton, 1986). Previously, Lana et al. (1998) reported that forage- and concentrate-fed cattle demonstrate different populations of ammonia-producing bacteria in the rumen and that optimal ammonia concentration in rumen fluid results in the maximum fermentation rate and production of microbial protein (Mehrez et al., 1977). In our study, the remarkable increase in $\mathrm{NH}_{3}-\mathrm{N}$ concentration in the third and fourth challenges (up to 20.0 and $18.7 \mathrm{mg} / \mathrm{dL}$, respectively) may represent a simultaneous increase in microbial protein production, and the $\mathrm{NH}_{3}-\mathrm{N}$ concentration detected was close to optimal ammonia concentration (235 or more mg/L; Mehrez et al., 1977) for maximal rate of fermentation. Therefore, the increase in total VFA during the HG period of the later challenges may be associated with the increase in $\mathrm{NH}_{3}-\mathrm{N}$ concentration and support our hypothesis that bacterial adaptation in fermentation occurred during repeated SARA challenges. However, the dynamics of ammonia in the rumen by ruminal bacteria, efflux to the omasum, or absorption across the ruminal wall were not determined in this study, and further studies are required to clarify the utilization mechanisms of ammonia during repeated SARA challenges in Holstein cattle. In contrast with bacterial fermentative adaptation, total accumulated VFA and low lactic acid concentration in the rumen fluid were reported to be features of adapted rumen parameters to concentrate diet (Lana et al., 1998). This may have caused the weak diurnal changes in ruminal $\mathrm{pH}$ during the HG period of the fourth challenge in our study.

Previously, Firmicutes and Bacteroidetes phyla were identified as the rumen core microbiome (Petri et al., 2013; Henderson et al., 2015). In other words, these 2 phyla may be less affected by changes in the rumen environment, such as dietary changes or acidic challenge. Several studies have reported that the proportion of Bacteroidetes decreases during high-grain diets due to the high acidity in the rumen (Mao et al., 2013; Sato, 2016). For instance, Mao et al. (2013) reported that diets that induced SARA in dairy cattle lead to significantly reduced ruminal $\mathrm{pH}$, increased proportion of Firmicutes (47.2-56.9\%), and decreased relative abundance of Bacteroidetes (44.4-34.2\%) compared with control diets. Although a decreased proportion of Bacteroidetes and an increased proportion of Firmicutes were identified during the HG period compared with the HF period in the first and fourth challenges, the degree of fluctuation in the relative abundance of these phyla was less drastic in the fourth challenge compared with the first challenge. This observation was consistent with the alleviation of adverse changes in ruminal $\mathrm{pH}$ during the fourth challenge. Therefore, it can be suggested that the rumen bacterial phyla adapted to the repeated SARA challenges, concurrent with ruminal $\mathrm{pH}$ regulation. However, due to the highly diverse bacteria found within a phylum, only limited conclusions about 
features of certain taxa within a certain phylum can be made (Wetzels et al., 2017).

Petri et al. (2013) also suggested that the rumen core microbiome is remarkably stable when diet is transitioned from forage to concentrate or during acidic challenge and recovery and that Prevotella $(22.2 \%)$ was the only genus that was present in all 8 heifers across all diets (Petri et al., 2013). Prevotella bacteria can dominate in the rumen under a range of diets due to its ability to use a variety of substrates (Golder et al., 2014) and was identified as the most predominant genus in our study. Moreover, the low relative abundance of Prevotella during the HG period was consistent with a previous study that reported that starch addition reduced the relative abundance of Prevotella (Zened et al., 2013). Although the Ruminococcus genus is well known for largely comprising cellulolytic bacteria, Ruminococcus bromii, which can ferment starch, was a dominant bacterial population in the rumen (Klieve et al., 2007). Therefore, an increase in starch-fermenting Ruminococcus species may have contributed to the higher relative abundance of Ruminococcus observed during the HG period in this study. Eubacterium is associated with hemicellulolytic activity in the rumen (Taguchi et al., 2004), and the relative abundance of Eubacterium was found to be higher during the HF period than during the HG period. The Oscillibacter genus belonging to the Firmicutes phylum largely comprises fibrolytic bacteria (Thoetkiattikul et al., 2013). Studies have shown that Oscillibacter spp. in the rumen has an optimal growth rate at around $\mathrm{pH} 6.0$ (Iino et al., 2007; Lee et al., 2013); we found that the relative abundance of Oscillibacter was significantly increased during the HF period. Collectively, alterations in the relative abundance of ruminal genera were associated with substrates necessary for bacterial growth and changes in ruminal $\mathrm{pH}$. These changes may influence the bacterial community structure and fermentation in the rumen.

Several studies were conducted to investigate the effect of SARA challenge on the adaptation and recovery of rumen bacteria during acidic challenges (Hook et al., 2011; McCann et al., 2016; Plaizier et al., 2017). In this study, we identified a 37-genera core bacterial community shared by all cattle regardless of the diet and challenge. Among these, several genera (Clostridium, Eubacterium, Oscillibacter, and unclassified Clostridiaceae) were affected by the challenge, and the relative abundance of Clostridium and Butyrivibrio showed similar increases during the HG period of the first challenge, although statistical significance was not reached in the Butyrivibrio. Hook et al. (2011) reported that Clostridium spp. and Butyrivibrio spp. were detected during the baseline (hay diet), high-grain diet (SARA challenge), and recovery (hay diet) periods, whereas bacterium Eubacterium spp. was not identified during the high-grain diet. Moreover, a slight increase in the number of Clostridium spp. and Butyrivibrio spp. during the SARA challenge was identified; this number decreased during the recovery period (Hook et al., 2011). This was consistent with the higher relative abundance of Clostridium and Butyrivibrio during the HG period. However, the adaptation and recovery mechanisms of bacterial community to repeated SARA challenges are largely unknown due to the limited information on rumen bacterial communities during the second and third challenges. Therefore, it is necessary to clarify the longitudinal adaptation and recovery mechanisms of rumen bacteria.

Although 16S reads were clustered using a 96\% pairwise identity cutoff for OTU analysis to reduce overestimation of species richness, sequences were clustered independently into OTU with a cutoff value of $97 \%$ similarity for bacterial diversity analysis to identify bacterial species-level OTU. Therefore, resulting OTU will be different between the 2 different clustering steps and should be considered with care. In our study, bacterial diversity indices were found to be higher during the HF period than during the HG period in both challenges. Interestingly, bacterial diversity indices during the $\mathrm{HF}$ period of the fourth challenge showed the highest values. In addition, bacterial diversity of the fourth challenge was higher during the HG period than during the HF period of the first challenge, which was consistent with rarefaction curve results. A severe decrease in diversity was also reported in previous studies when cattle were suffering from SARA or ruminal acidosis (Mao et al., 2013; Petri et al., 2013). Mao et al. (2013) reported that SARA-inducing diets reduced ruminal $\mathrm{pH}$ and bacterial diversity. Moreover, significantly decreased OTU and Shannon indices by high-grain diets can be recovered to prechallenge levels during the recovery period (Hook et al., 2011). Although bacterial community and diversity during the second and third challenges were not investigated in the present study, bacterial diversity in the fourth challenge (HG period) was higher than that during baseline (HF period of first challenge). Furthermore, principal coordinates analysis plots during the HG period exhibited dispersed distribution and showed low similarity from the HF period in both challenges, suggesting that the bacterial community during the later challenges developed its own unique rumen bacterial community structure. Therefore, these results suggested that bacterial diversity and similarity are influenced by dietary transitions and repeated SARA challenges and may be associated with reduced adverse changes in ruminal $\mathrm{pH}$ during the last challenge. 


\section{CONCLUSIONS}

To our knowledge, this is the first study that reports on changes in ruminal $\mathrm{pH}$ and adaptations of ruminal bacterial composition, diversity, and similarity in Holstein bulls during repeated SARA induction. The severity of SARA in the second challenge was greater compared with that in first challenge but was alleviated in the third and fourth challenges. We identified 37 genera as rumen core bacteria, some of which were affected by dietary transition (Prevotella, Ruminococcus, Eubacterium, and Oscillibacter) and repeated SARA challenges (Clostridium, Eubacterium, and unclassified Clostridiaceae). Bacterial diversity was higher in the fourth challenge compared with the first challenge. Bacterial communities during the $\mathrm{HG}$ period in the first and fourth challenges showed stark contrast with each other and with those from the HF period. These results supported our hypothesis that repeated SARA challenges induce adverse changes in ruminal $\mathrm{pH}$ and that the bacterial community can adapt to these changes by altering their composition, diversity, and similarity. This study provided novel insight into the effect of SARA challenges on ruminal $\mathrm{pH}$, fermentation, and bacterial community in Holstein bulls.

\section{ACKNOWLEDGMENTS}

This work was supported by the Cabinet Office, Government of Japan, Cross-Ministerial Strategic Innovation Promotion Program (SIP) "Technologies for Creating Next-Generation Agriculture, Forestry and Fisheries."

\section{REFERENCES}

Bevans, D. W., K. A. Beauchemin, K. S. Schwartzkopf-Genswein, J. J. McKinnon, and T. A. McAllister. 2005. Effect of rapid or gradual grain adaptation on subacute acidosis and feed intake by feedlot cattle. J. Anim. Sci. 83:1116-1132.

Burrin, D. G., and R. A. Britton. 1986. Response to monensin in cattle during subacute acidosis. J. Anim. Sci. 63:888-893.

DeVries, T. J., K. A. Beauchemin, F. Dohme, and K. S. SchwartzkopfGenswein. 2009. Repeated ruminal acidosis challenges in lactating dairy cows at high and low risk for developing acidosis: Feeding, ruminating, and lying behavior. J. Dairy Sci. 92:5067-5078.

Dohme, F., T. J. DeVries, and K. A. Beauchemin. 2008. Repeated ruminal acidosis challenges in lactating dairy cows at high and low risk for developing acidosis: Ruminal pH. J. Dairy Sci. 91:35543567 .

Golder, H. M., S. E. Denman, C. McSweeney, P. Celi, and I. J. Lean. 2014. Ruminal bacterial community shifts in grain-, sugar-, and histidine-challenged dairy heifers. J. Dairy Sci. 97:5131-5150.

Gozho, G. N., J. C. Plaizier, D. O. Krause, A. D. Kennedy, and K. M. Wittenberg. 2005. Subacute ruminal acidosis induces ruminal lipopolysaccharide endotoxin release and triggers an inflammatory response. J. Dairy Sci. 88:1399-1403.

Henderson, G., F. Cox, S. Ganesh, A. Jonker, W. Young, G. R. C. Collaborators, and P. H. Janssen. 2015. Rumen microbial community composition varies with diet and host, but a core microbiome is found across a wide geographical range. Sci. Rep. 5:14567.

Hook, S. E., M. A. Steele, K. S. Northwood, J. Dijkstra, J. France, A. D. G. Wright, and B. W. McBride. 2011. Impact of subacute ruminal acidosis (SARA) adaptation and recovery on the density and diversity of bacteria in the rumen of dairy cows. FEMS Microbiol. Ecol. 78:275-284.

Iino, T., K. Mori, K. Tanaka, K. I. Suzuki, and S. Harayama. 2007. Oscillibacter valericigenes gen. nov., sp. nov., a valerate-producing anaerobic bacterium isolated from the alimentary canal of a Japanese corbicula clam. Int. J. Syst. Evol. Microbiol. 57:1840-1845.

Kim, S. W., W. Suda, S. Kim, K. Oshima, S. Fukuda, H. Ohno, H. Morita, and M. Hattori. 2013. Robustness of gut microbiota of healthy adults in response to probiotic intervention revealed by high-throughput pyrosequencing. DNA Res. 20:241-253.

Kim, Y.-H., R. Nagata, N. Ohtani, T. Ichijo, K. Ikuta, and S. Sato. 2016. Effects of dietary forage and calf starter diet on ruminal pH and bacteria in Holstein calves during weaning transition. Front. Microbiol. 7:1575.

Kimura, A., S. Sato, T. Kato, K. Ikuta, N. Yamagishi, K. Okada H. Mizuguchi, and K. Ito. 2012. Relationship between pH and temperature in the ruminal fluid of cows, based on a radio-transmission pH-measurement system. J. Vet. Med. Sci. 74:1023-1028.

Klieve, A. V., M. N. O'Leary, L. McMillen, and D. Ouwerkerk. 2007. Ruminococcus bromii, identification and isolation as a dominant community member in the rumen of cattle fed a barley diet. J. Appl. Microbiol. 103:2065-2073.

Lana, R. P., J. B. Russell, and M. E. Van Amburgh. 1998. The role of $\mathrm{pH}$ in regulating ruminal methane and ammonia production. J. Anim. Sci. 76:2190-2196.

Lee, G. H., M. S. Rhee, D. H. Chang, J. Lee, S. Kim, M. H. Yoon, and B. C. Kim. 2013. Oscillibacter ruminantium sp. nov., isolated from the rumen of Korean native cattle. Int. J. Syst. Evol. Microbiol. 63:1942-1946.

Lozupone, C., and R. Knight. 2005. UniFrac: A new phylogenetic method for comparing microbial communities. Appl. Environ. Microbiol. 71:8228-8235.

Mao, S. Y., R. Y. Zhang, D. S. Wang, and W. Y. Zhu. 2013. Impact of subacute ruminal acidosis (SARA) adaptation on rumen microbiota in dairy cattle using pyrosequencing. Anaerobe 24:12-19.

McCann, J. C., S. Luan, F. C. Cardoso, H. Derakhshani, E. Khafipour, and J. J. Loor. 2016. Induction of subacute ruminal acidosis affects the ruminal microbiome and epithelium. Front. Microbiol. 7:701.

Mehrez, A. Z., E. R. Ørskov, and I. McDonald. 1977. Rates of rumen fermentation in relation to ammonia concentration. Br. J. Nutr. 38:437-443.

Metzler-Zebeli, B. U., A. Khol-Parisini, L. Gruber, and Q. Zebeli. 2015. Microbial populations and fermentation profiles in rumen liquid and solids of Holstein cows respond differently to dietary barley processing. J. Appl. Microbiol. 119:1502-1514.

Nagaraja, T. G., and E. C. Titgemeyer. 2007. Ruminal acidosis in beef cattle: The current microbiological and nutritional outlook. J. Dairy Sci. 90(Suppl. 1):E17-E38.

Owens, F. N., D. S. Secrist, W. J. Hill, and D. R. Gill. 1998. Acidosis in cattle: A review. J. Anim. Sci. 76:275-286.

Penner, G. B., K. A. Beauchemin, and T. Mutsvangwa. 2007. Severity of ruminal acidosis in primiparous Holstein cows during the periparturient period. J. Dairy Sci. 90:365-375.

Petri, R. M., P. Pourazad, R. Khiaosa-Ard, F. Klevenhusen, B. U. Metzler-Zebeli, and Q. Zebeli. 2017. Temporal dynamics of in situ fiber-adherent bacterial community under ruminal acidotic conditions determined by $16 \mathrm{~S}$ rRNA gene profiling. PLoS One 12:e0182271.

Petri, R. M., T. Schwaiger, G. B. Penner, K. A. Beauchemin, R. J. Forster, J. J. McKinnon, and T. A. McAllister. 2013. Characterization of the core rumen microbiome in cattle during transition from forage to concentrate as well as during and after an acidotic challenge. PLoS One 8:e83424.

Plaizier, J. C., D. O. Krause, G. N. Gozho, and B. W. McBride. 2008. Subacute ruminal acidosis in dairy cows: The physiological causes, incidence and consequences. Vet. J. 176:21-31. 
Plaizier, J. C., S. Li, A. M. Danscher, H. Derakshani, P. H. Andersen, and E. Khafipour. 2017. Changes in microbiota in rumen digesta and feces due to a grain-based subacute ruminal acidosis (SARA) challenge. Microb. Ecol. 74:485-495.

Pourazad, P., R. Khiaosa-Ard, M. Qumar, S. U. Wetzels, F. Klevenhusen, B. U. Metzler-Zebeli, and Q. Zebeli. 2016. Transient feeding of a concentrate-rich diet increases the severity of subacute ruminal acidosis in dairy cattle. J. Anim. Sci. 94:726-738.

Pruesse, E., C. Quast, K. Knittel, B. M. Fuchs, W. Ludwig, J. Peplies, and F. O. Glöckner. 2007. SILVA: A comprehensive online resource for quality checked and aligned ribosomal RNA sequence data compatible with ARB. Nucleic Acids Res. 35:7188-7196.

Rabelo, E., R. L. Rezende, S. J. Bertics, and R. R. Grummer. 2003. Effects of transition diets varying in dietary energy density on lactation performance and ruminal parameters of dairy cows. J. Dairy Sci. 86:916-925.

Sato, S. 2016. Pathophysiological evaluation of subacute ruminal acidosis (SARA) by continuous ruminal $\mathrm{pH}$ monitoring. Anim. Sci. J. 87:168-177.

Sato, S. H. Mizuguchi, K. Ito, K. Ikuta, A. Kimura, and K. Okada 2012. Technical note: Development and testing of a radio transmission $\mathrm{pH}$ measurement system for continuous monitoring of ruminal $\mathrm{pH}$ in cows. Prev. Vet. Med. 103:274-279.

Schloss, P. D., D. Gevers, and S. L. Westcott. 2011. Reducing the effects of PCR amplification and sequencing artifacts on 16S rRNAbased studies. PLoS One 6:e27310.

Schloss, P. D., S. L. Westcott, T. Ryabin, J. R. Hall, M. Hartmann, E. B. Hollister, A. L. Ryan, B. O. Brian, H. P. Donovan, J. R Courtney, W. S. Jason, S. Blaz, G. T. Gerhard, J. V. H. David, and F. W. Carolyn. 2009. Introducing mothur: open-source, platform-independent, community-supported software for describing and comparing microbial communities. Appl. Environ. Microbiol. 75:7537-7541

Taguchi, H., S. Koike, Y. Kobayashi, I. K. Cann, and S. Karita. 2004. Partial characterization of structure and function of a xylanase gene from the rumen hemicellulolytic bacterium Eubacterium ruminantium. Anim. Sci. J. 75:325-332.

Thoetkiattikul, H., W. Mhuantong, T. Laothanachareon, S. Tangphatsornruang, V. Pattarajinda, L. Eurwilaichitr, and V. Champreda 2013. Comparative analysis of microbial profiles in cow rumen fed with different dietary fiber by tagged $16 \mathrm{~S}$ rRNA gene pyrosequencing. Curr. Microbiol. 67:130-137.

Wetzels, S. U., E. Mann, P. Pourazad, M. Qumar, B. Pinior, B. U. Metzler-Zebeli, M. Wanger, S. Schmitz-Esser, and Q. Zebeli. 2017. Epimural bacterial community structure in the rumen of Holstein cows with different responses to a long-term subacute ruminal acidosis diet challenge. J. Dairy Sci. 100:1829-1844.

Zebeli, Q., M. Tafaj, I. Weber, H. Steingass, and W. Drochner. 2008. Effects of dietary forage particle size and concentrate level on fermentation profile, in vitro degradation characteristics and concentration of liquid- or solid-associated bacterial mass in the rumen of dairy cows. Anim. Feed Sci. Technol. 140:307-325.

Zened, A., S. Combes, L. Cauquil, J. Mariette, C. Klopp, O. Bouchez, A. Troegeler-Meynadier, and F. Enjalbert. 2013. Microbial ecology of the rumen evaluated by 454 GS FLX pyrosequencing is affected by starch and oil supplementation of diets. FEMS Microbiol. Ecol. 83:504-514 